\title{
Electron probing of the oxygen evolving $\mathrm{Ba}_{0.5} \mathrm{Sr}_{0.5} \mathrm{Co}_{0.8} \mathrm{Fe}_{0.2} \mathrm{O}_{3-\delta}$
}

\section{Tzu-Hsien Shen ${ }^{1}$, Liam Spillane ${ }^{2}$, Jan Vavra ${ }^{1}$ and Vasiliki Tileli ${ }^{1}$}

${ }^{1}$ Institute of Materials, École polytechnique fédérale de Lausanne, Lausanne, Switzerland, ${ }^{2}$ Gatan Inc., Pleasanton, California, United States

Electron characterization of high-performing catalysts is essential for recognizing the attributes that are responsible for their activity. For example, our recent transmission electron microscopy (TEM) study on $\mathrm{Ba} 0.5 \mathrm{Sr} 0.5 \mathrm{Co} 0.8 \mathrm{Fe} 0.2 \mathrm{O} 3-\delta(\mathrm{BSCF})$, an oxygen evolving perovskite that exhibits excellent activity [1], has revealed a spinel surface structure that accommodates the conversion to the active oxyhydroxide phase during cyclic voltammetry (CV) [2]. To better understand the evolution of catalysts during the electrochemical processes, herein, we report on morphological changes of BSCF particles using identical location scanning TEM (STEM) [3] and formation of products using operando electron energy-loss spectroscopy (EELS) in liquid phaseTEM [4].

In-house fabricated microelectromechanical system (MEMS) Si chips were used for the experiments. Figure 1a depicts the electrochemical chip patterned with a glassy carbon thin film working electrode for the identical location measurements. The MEMS chip with loaded BSCF particles was initially used to characterize the BSCF particles of interest. After initial TEM analysis, a solution of $0.1 \mathrm{M} \mathrm{KOH}$ was used as the electrolyte for the $\mathrm{CV}$ measurements, Figure 1b. The same particle was then reanalyzed. Figure 1c shows high-angle annular dark-field STEM (HAADF-STEM) images of the same BSCF particle at different CV cycling conditions. As the number of cycles increases, the particle becomes porous. This porosity could be e-beam induced degradation of a weak underlying lattice induced by the reversible transformation of the oxyhydroxide phase during electrochemical cycling. Additionally, this porosity can be associated with a lower oxygen evolution reaction (OER) take-off potential as seen in the CV curves. A porous surface network could expose more active sites and, hence, lead to an enhancement of the activity of the catalysts for the first $10 \mathrm{CV}$ cycles that were probed, in line with previous reports [5].

To further investigate the molecular oxygen evolution of single BSCF particles, electrochemical liquid-phase STEM-EELS was performed in real-time. In this case, the BSCF particles were dropcasted on a MEMS chip patterned with three Pt thin film electrodes, a working (WE), a reference (RE), and a counter electrode (CE), as shown in Figure 2a. The thickness of the Pt layer was kept as thin as possible $(\sim 15 \mathrm{~nm})$ for the sensitive-tothickness nanoanalytical EELS experiments. The MEMS chip together with a spacer chip were assembled and the electrolyte was injected into the cell. Figure 2b depicts the annular dark-field (ADF) image of the BSCF particles sitting on the Pt WE electrode inside the liquid cell. The electron beam was placed in proximity to the BSCF particles (red point in Figure 2b) while avoiding being placed directly on the particles to avoid ebeam induced artifacts during probing. Potential cycling was applied while EEL spectra of O K edge were acquired in spot mode. The molecular oxygen peak feature, which is the asterisk at energy-loss $531 \mathrm{eV}$ in Figure $2 \mathrm{c}$, becomes pronounced when the anodic applied potential increases above a threshold. Thus, the evolution of the $\mathrm{O} 2$ peak feature confirms the evolution of molecular oxygen from single BSCF particles during CV cycling.

In conclusion, our work on the identical surface probing of BSCF and the real-time EELS analysis open a new pathway for catalyst characterization, aiding the future development of catalysts. 
a

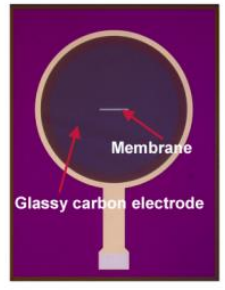

b

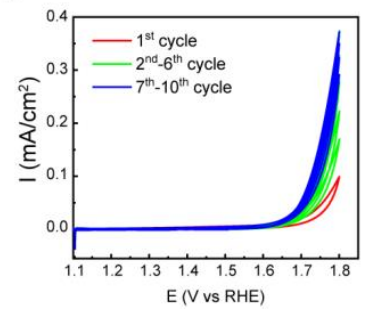

c

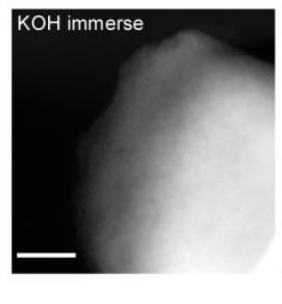

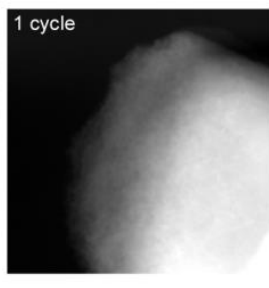
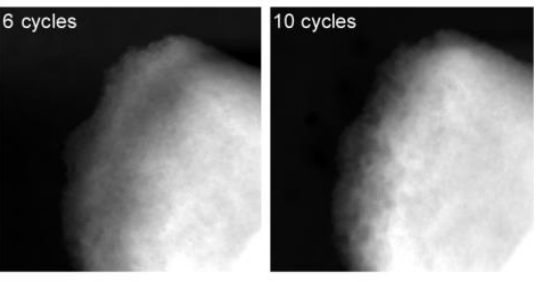

Figure 1. Identical STEM characterization of BSCF under potential cycling. (a) MEMS electrochemical chip with a glassy carbon working electrode. (b) Polarization curves of 10 cycles. (c) Morphological evolution of the same BSCF particle after certain CV cycles. The scale bar is $50 \mathrm{~nm}$.
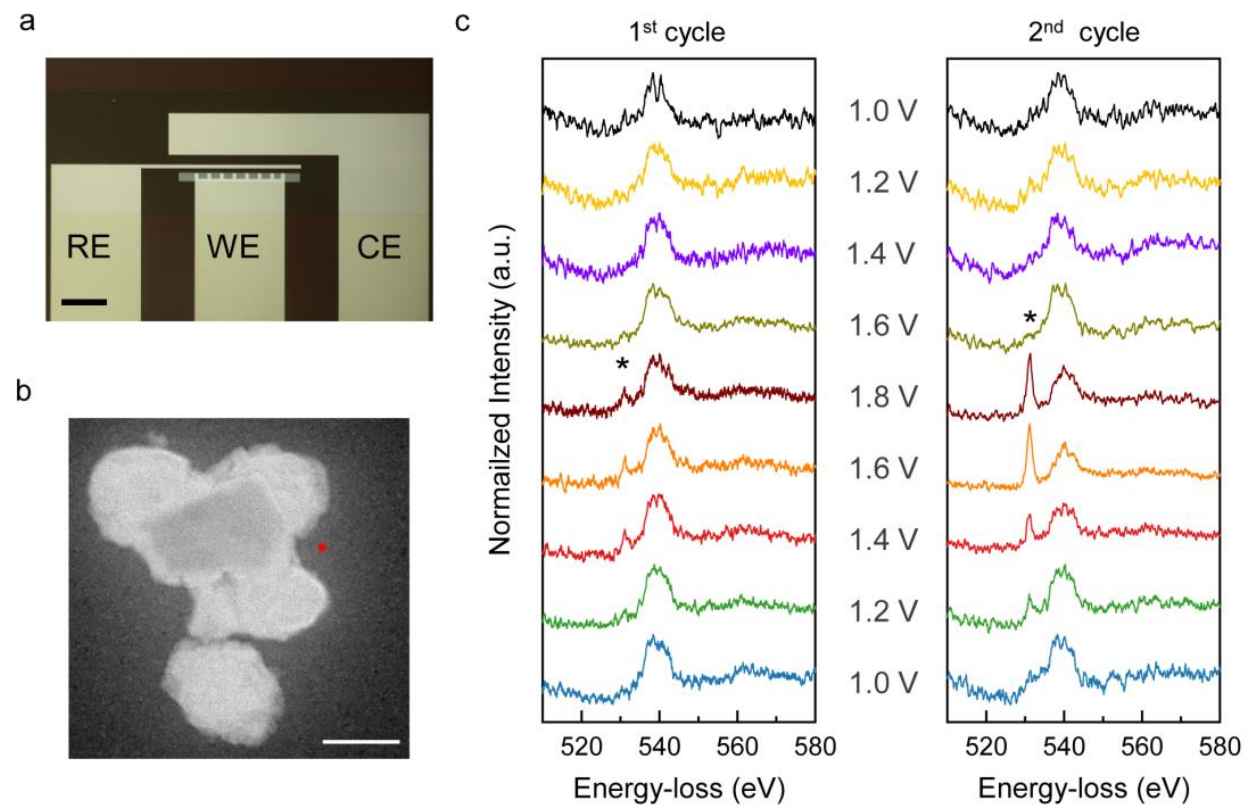

Figure 2. EELS analysis of molecular oxygen evolution near BSCF. (a) MEMS electrochemical chip patterned with three Pt electrodes. The scale bar is $250 \mu \mathrm{m}$. (b) HAADF-STEM image of BSCF particles in the liquid cell. The red point indicates the electron beam position for the acquisition of the EEL spectra. The scale bar is $400 \mathrm{~nm}$. (c) Potential dependent O K EEL spectra for the first $2 \mathrm{CV}$ cycles. The peak feature indicated by the asterisks at $531 \mathrm{eV}$ is the fingerprint of molecular oxygen evolving during cycling.

\section{References}

[1] J. Suntivich, K. J. May, H. a Gasteiger, J. B. Goodenough, and Y. Shao-horn, Science (80-. ). 334, 1383 (2011).

[2] T. H. Shen, L. Spillane, J. Vavra, T. H. M. Pham, J. Peng, Y. Shao-Horn, and V. Tileli, J. Am. Chem. Soc. 142,15876 (2020).

[3] F. Ruiz-Zepeda, M. Gatalo, A. Pavlišič, G. Dražić, P. Jovanovič, M. Bele, M. Gaberšček, and N. Hodnik, Nano Lett. 19, 4919 (2019).

[4] N. De Jonge and F. M. Ross, Nat. Nanotechnol. 6, 695 (2011).

[5] M. Risch, A. Grimaud, K. J. May, K. A. Stoerzinger, T. J. Chen, A. N. Mansour, and Y. Shao-Horn, J. Phys. Chem. C 117, 8628 (2013). 